\title{
EVOLUTIONARY STATUS OF MASSIVE ALGOLS - A REVISION OF CLASSICAL THEORY
}

\author{
J. ZIOXK OW S I \\ Institute of Astronomy, Polish Academy of Sciences, Warsaw, Poland
}

(Read by B. Paczynski)

\begin{abstract}
The evolutionary status of Algol systems is examined.
There is a growing suspicion (Plavec, 1973) or even conviction (Hall, 1975) that contrary to the classical theory (Plavec, 1968; Paczynski, 1971) some or even most of the best known Algol-type systems represent Case B rather than Case A of binary evolution. In Figure 1 we present the initial periods of massive algols against the total mass of the system assuming the conservation of total mass and total orbital angular momentum. It seems that at least half of these systems represent Case B. Unfortunately, the classification of the individual systems is still highly unreliable, as is most dramatically illustrated by the recent history of such a well known binary as U Cep. Only for a very few systems ( $\beta$ Lyr, SV Cen, U Cep) do consistent data concerning the absolute parameters of the system, the properties of secondary component and the rate of the period change permit a sure classification. Using more relaxed but still justifiable criteria we may state that systems $\beta \mathrm{Lyr}$, V367 Cyg, V453 Sco, U Cep, V356 Sgr and probably also KU Cyg and S Cnc evolve in Case B while SV Cen, V Pup and $\mu_{1}$ Sco evolve in Case A. The systems RS Vul; Z Vul and USge are likely candidates for Case B. All systems evolving in Case B have to be in the fast stage of evolution and so have to exhibit rapid period changes. For example, the system V356 Sgr ( $P=8.9$ days), for which there are no observational reports about any period changes, should increase its period at the rate of about $1 \mathrm{~s}$ per cycle on the basis of the general theory. We should ask the observers to confirm or to refute this prediction.

Considering the ratio of the evolutionary lifetimes in Case A and Case B (over 100) the apparent deficit of binaries evolving in Case A seems to be indirect evidence that most of the binaries evolving in Case $A$ end up as configurations with thick common envelope and are not recognised as Algol-type systems. The contact binary SV Cen is probably evolving in this direction.
\end{abstract}

\section{References}

Hall, D. S. : 1975, Acta Astron. 25, 1.

Plavec, M.: 1973, IAU Symp. 51, 216.

Plavec, M.: 1968, Advan. Astron. Astrophys. 6, 201.

Paczynski, B.: 1971, Ann. Rev. Astron. Astrophys. 9, 183. 


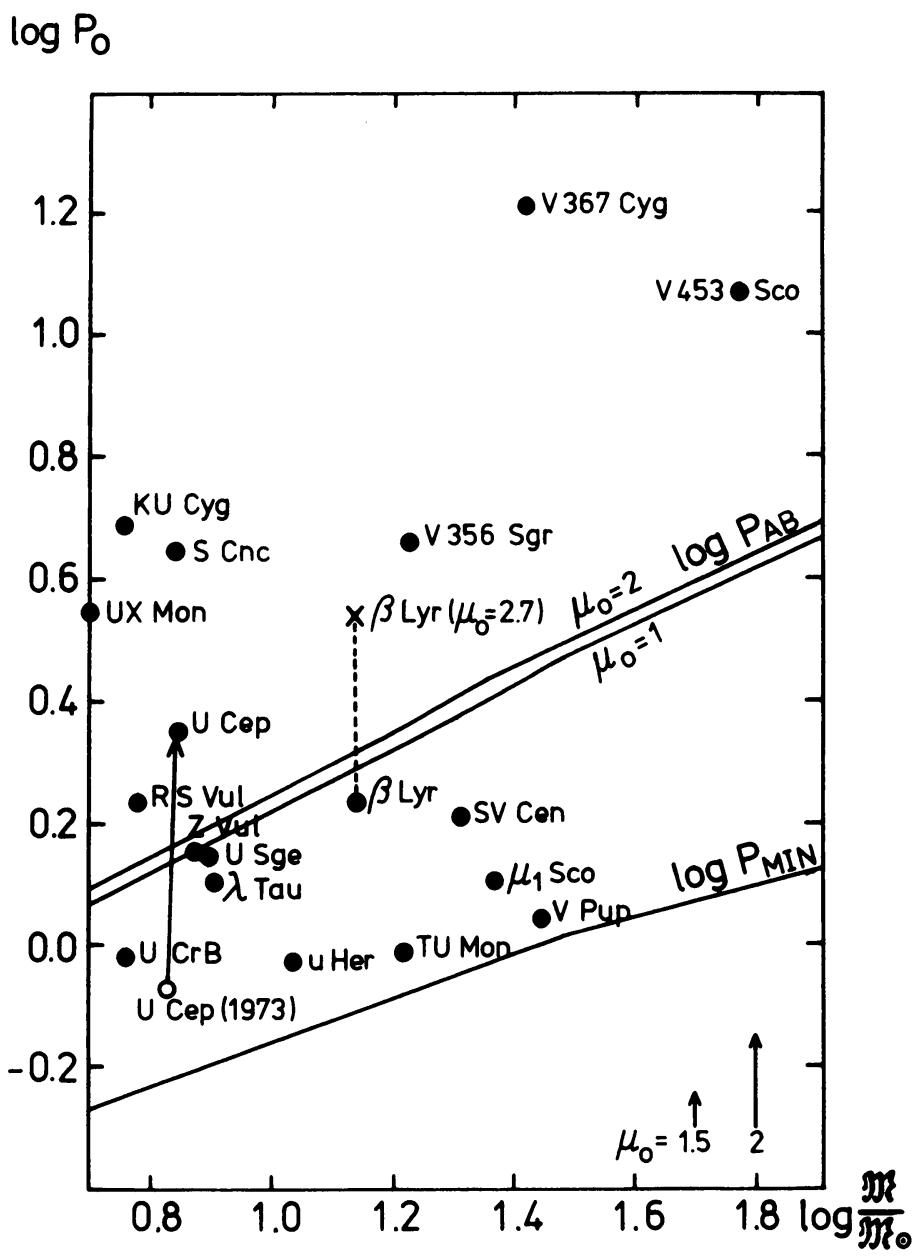

Fig. 1. Positions of all systems are shown assuming an initial mass ratio $\mu_{0}=1$. For initial mass ratios higher than 1 all positions should be lifted up by the amounts indicated at the lower right. 